\title{
Characteristics and Operation Conditions of a Closed-Field Unbalanced Dual Magnetrons Plasma Sputtering System
}

\author{
Bahaa T. Chiad1, Mohammad K. Khalaf', Firas J. Kadhim¹, Oday A. Hammadi ${ }^{*}$ \\ ${ }^{1}$ Department of Physics, College of Science, University of Baghdad, Baghdad, Iraq \\ ${ }^{2}$ Center of Applied Physics, Ministry of Science and Technology, Baghdad, Iraq \\ ${ }^{3}$ Department of Physics, College of Education, Al-Iraqia University, Baghdad, Iraq \\ Email: *oday.hamadi@yahoo.com
}

Received 15 April 2014; revised 27 May 2014; accepted 17 July 2014

Copyright (C) 2014 by authors and OALib.

This work is licensed under the Creative Commons Attribution International License (CC BY).

http://creativecommons.org/licenses/by/4.0/

(c) (i) Open Access

\begin{abstract}
In this work, a home-made closed-field unbalanced magnetron system for plasma sputtering purposes was constructed and operated. The effect of magnetron was introduced by comparing the obtained Paschen's curve in existence of magnetron with and without magnetron. Characterization of Paschen's curve as well as discharge current with gas pressure at different distances between the discharge electrodes was introduced. Optimum conditions to operate such home-made system for sputtering purpose were determined.
\end{abstract}

Keywords

Physical Vapor Deposition, Sputtering, Magnetron Sputtering, Paschen's Law

Subject Areas: Applied Physics, Plasma

\section{Introduction}

A glow discharge is simply produced by applying an electric potential on a gas sample between two electrodes placed inside a vacuum chamber [1]. This glow discharge is a common source of plasma that can be established through an avalanche like ionization of gas neutrals at specific conditions for gas pressure and applied voltage [2] [3].

At low kinetic energies (energies between 0 and about $50 \mathrm{eV}$ ), the ion does not have sufficient energy to dislodge the target atoms and thus the ejection of target particles occurs only for very special collision geometries [4]. With moderate energies (between 50 and roughly $1 \mathrm{keV}$ ), the ions impact dislodge "knock-on" atoms into

${ }^{*}$ Corresponding author.

How to cite this paper: Chiad, B.T., Khalaf, M.K., Kadhim, F.J. and Hammadi, O.A. (2014) Characteristics and Operation Conditions of a Closed-Field Unbalanced Dual Magnetrons Plasma Sputtering System. Open Access Library Journal, 1: e650. http://dx.doi.org/10.4236/oalib.1100650 
the target, which by their turn will dislodge other target atoms [5]. Several studies showed that the ion energies must exceed four times the binding energy of the atoms of the target surface to induce sputtering [6]-[9]. This induces a collision cascade that eject atoms, ions, electrons and neutrals from the first 10 to $50 \AA$ of the surface of a target [7].

Initially existence of electrons is a requirement for this ionization process and such electrons, which may normally exist by cosmic radiation, are accelerated by the applied electric field towards the anode and consequently gained sufficient energy to collide with gas atoms and ionize them [10]-[12]. Hence, the number of electrons existing between the two electrodes will be multiplied at a rate described by the Townsend's first ionization coefficient, which represents the relative increase in electron flux per unit path length. The continuous electron production is required to sustain the discharge and provided by the production of secondary electrons induced during the ion impact at the cathode [13].

The breakdown voltage depends on the product of pressure $(p)$ and electrode separation $(d)$ as this product is denoted as " $p d$ ", while this voltage weakly depends on the cathode material that defines the emission coefficient of secondary electrons. As well, the breakdown voltage is proportional to the product $p d$ at large values of this product and the electric field $(E=V / d)$ is scaled linearly with the pressure [14]. In case of small values of the product $p d$, only few collisions occur and higher voltage is applied to increase the probability of breakdown per collision. Hence, the minimum voltage required to ignite the discharge of a gas sample of pressure $p$ over a distance $d$ is defined at the minimum of Paschen's curve, where

$$
\left.p d\right|_{V_{\min }}=\frac{1}{A} \log \left(1+\frac{1}{\gamma_{e}}\right)
$$

If the pressure and/or separation distance is too large, ions generated in the gas are slowed by inelastic collisions so that they strike the cathode with insufficient energy to produce secondary electrons. In most sputtering glow discharges, the discharge starting voltage is relatively high.

\section{Experiment}

The main parts of the plasma sputtering system are shown in Figure 1 and the closed-field unbalanced magnetron (CFUBM) was employed at the cathode electrode. Electrodes (anode and cathode) were made of stainless steel and each was a disk of $8 \mathrm{~cm}$ in diameter and $4 \mathrm{~mm}$ in thickness. Two annular concentric magnets were placedbehind each electrodeto form the magnetron configuration. The outer diameters of the two magnets were $8 \mathrm{~cm}$ and $4 \mathrm{~cm}$, while the inner diameters were $4 \mathrm{~cm}$ and 3.2, respectively. The electrodes were connected to a DC power supply to provide the electrical power required for discharge. The lower electrode (anode) could be move vertically with respect to the fixed upper electrode (cathode) to adjust the separation of the two electrodes from 1 to $8 \mathrm{~cm}$.

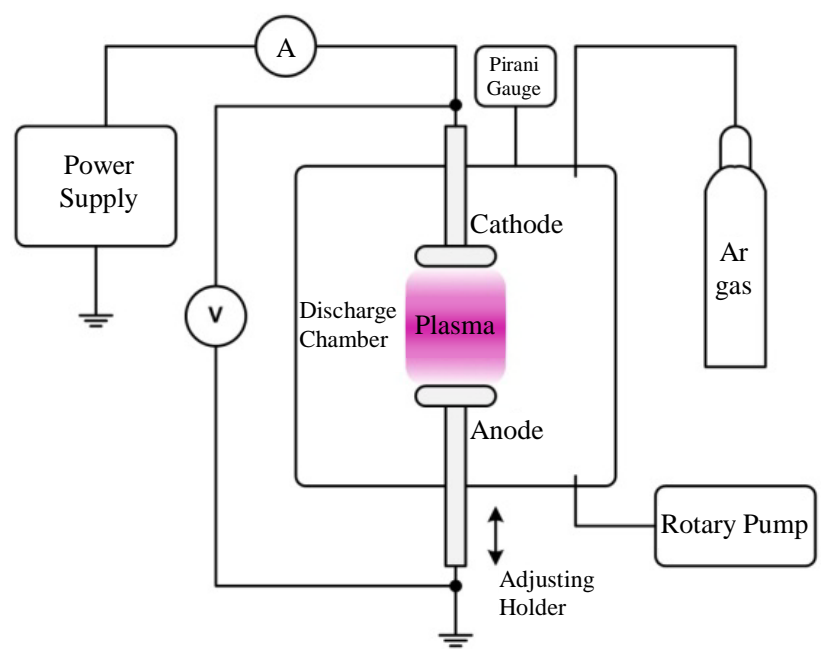

Figure 1. Schematic diagram of the system used in this work. 
Pure argon gas was used to produce the discharge plasma. A DC power supply up to $5 \mathrm{kV}$ was used for electrical discharge between the electrodes and both breakdown voltage (up to $1 \mathrm{kV}$ ) and discharge current (up to $100 \mathrm{~mA}$ ) were monitored by two digital voltmeter and ammeter, respectively. A current limiting resistor of 6.75 $\mathrm{kW}$ was connected in series to the discharge circuit in order to control the current flowing in the circuit. The discharge chamber was evacuated by a two-stage Leybold-Heraeus rotary pump and the vacuum inside chamber was measured by Pirani gauge connected to a vacuum controller from Balzers VWS 120. Argon gas was supplied to the chamber through a fine-controlled needle valve $(0-160 \mathrm{ccm})$ to control the gas pressure inside the chamber.

\section{Results and Discussion}

Sputtering of a target atom is just one of the possible effects resulting from the surface ion bombardment. Aside from sputtering, the second important process is the emission of secondary electrons from the target surface, which play a fundamental role in keeping the sputtering process itself. Figure 2 shows Paschen's curve for both cases of using and not using the magnetron at the upper electrode (cathode). As clearly shown, the effect of using magnetron lies in decreasing the breakdown voltage to about $15 \%$ of its initial (maximum) value, while the minimum voltage was decreased to seventh its value in absence of magnetron. However, Paschen's curves of both cases are identical with different minima, as the values of " $p . d$ " product were 2.6 and 2.2 mbar.cm when no magnetron and magnetrons were used, respectively. Using magnetrons caused the minimum to be shifted downward due the effect of magnetic field in trapping electrons near the electrode and hence a smaller amount of gas is required to reach breakdown point.

In dc sputtering, the electrons that are ejected from the cathode are accelerated away from the cathode and are not efficiently used for sustaining the discharge [4]. To avoid this effect, a magnetic field is added to the dc sputtering system that can deflect the electrons to near the target surface, and with appropriate arrangement of the magnets, the electrons can be made to circulate on a closed path on the target surface. This high current of electrons creates high-density plasma, from which ions can be extracted to sputter the target material, producing a magnetron sputter configuration.

A disadvantage of the magnetron sputtering configuration is that the plasma is confined near the cathode and is not available to active reactive gases in the plasma near the substrate for reactive sputter deposition. This difficulty can be overcome using an unbalanced magnetron configuration, where the magnetic field is such that some electrons can escape from the cathode region [15]. An unbalanced magnetron (UBM) has a proper magnetic field configuration in which a finite degree of the field lines from the outer magnetic pole diverge to the substrate, though the rest of the lines finish on the inner pole behind the target. Sufficient plasma density and a positive ion current on a metallic substrate even at a large distance from the target can be achieved in the unbalanced magnetron as compared with the balanced one [16].

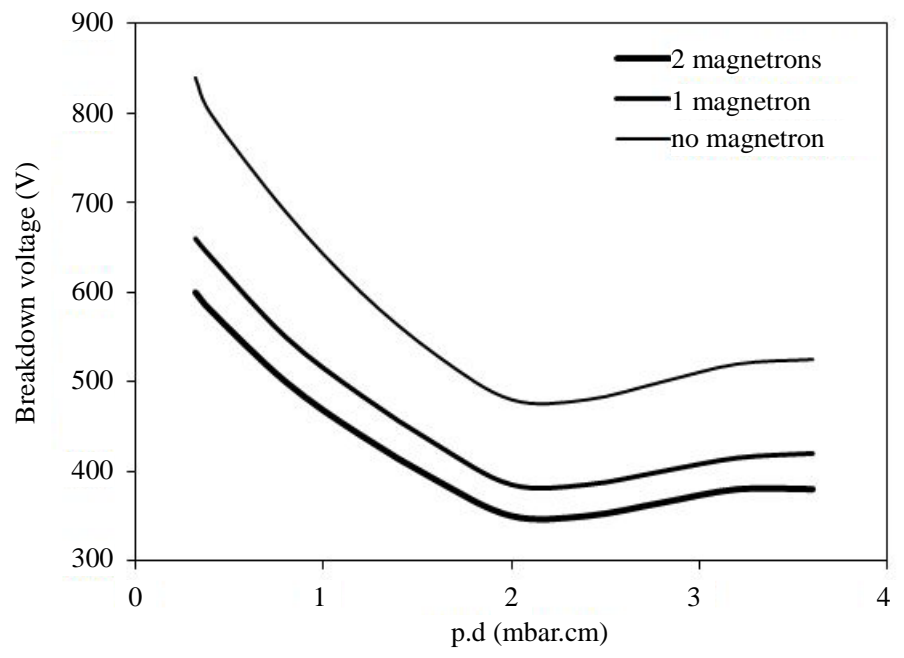

Figure 2. Paschen's curve for the constructed plasma sputtering system with and without magnetrons. 
The Paschen's curves of the plasma sputtering system in this work were plotted at different inter-electrode distances $(d)$ in presence of the magnetron at the cathode as shown in Figure 3. As shown, these curves are identical with the minimum shifted upward on the $p . d$ axis as the minimum voltage required to ignite the electrical discharge of the gas sample between the electrodes is increased with increasing the inter-electrode distance $(d)$.

The plasma sputtering system was then characterized by the relation of discharge current to the gas pressure inside the chamber at different inter-electrode distances, as shown in Figure 4. Again, all curves are identical with the discharge current shifted upward on the vertical axis. As the distance between the electrodes is decreased, the current density from electron current emitted from the cathode $j_{e}(0)$ is increased because less number of electrons are able to reach the anode and hence lower current flows. However, compensation is required between gas pressure and distance to work at a given discharge current before converting into decreasing current as saturation is reached.

Paschen's curves at distances $2 \mathrm{~cm} \leq d \leq 1.4 \mathrm{~cm}$ were plotted in the same way done before, as shown in Figure 5. These curves were slightly different from those plotted in Figure 3, where no minima were determined but instead the breakdown voltage was decreased with increasing the product $p . d$ until a constant value reached at $260-270 \mathrm{~V}$. This means that the operation of the magnetron plasma sputtering system may not be preferred at separating distance between the electrodes lower than $2 \mathrm{~cm}$. No evidence in the surveyed literature for this situation but we think that working at $<2 \mathrm{~cm}$ distances may produce low-quality structures when compared to those obtained when working at $>2 \mathrm{~cm}$.

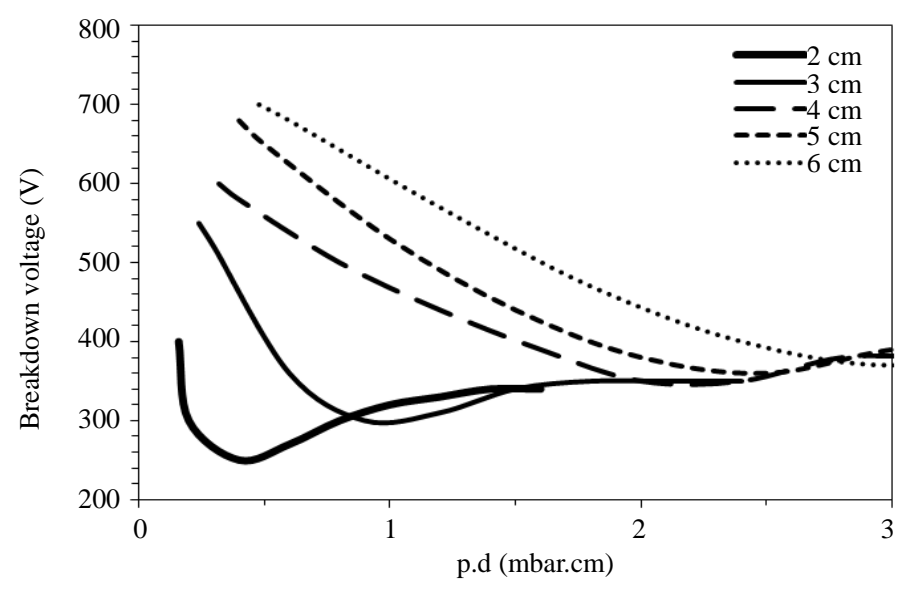

Figure 3. Paschen's curves for different separation distances $(d)$ between the discharge electrodes.

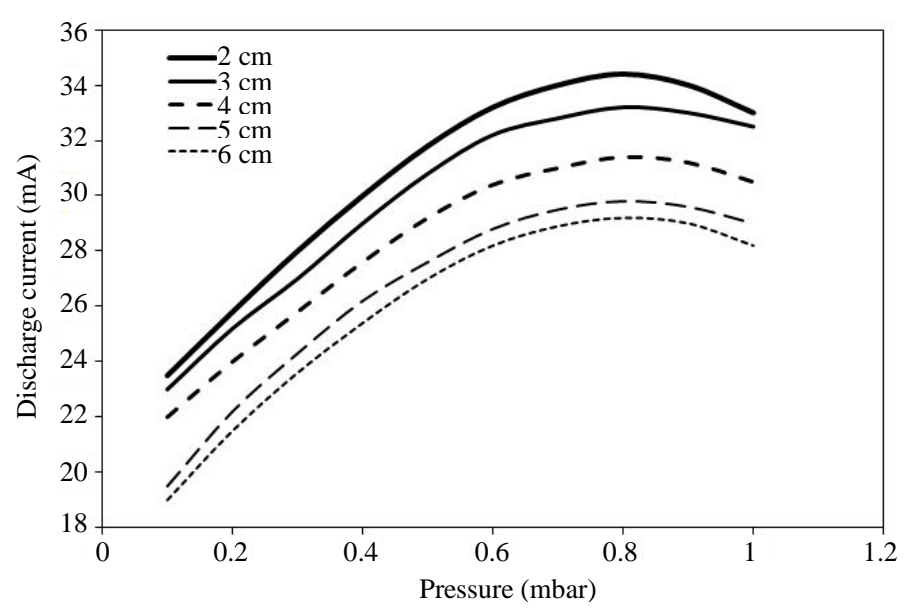

Figure 4. Variation of discharge current with increasing gas pressure for different inter-electrode distances $(d)$. 
By modeling the data obtained from this work, we plotted Paschen's curves for different separation distances between the discharge electrodes, as shown in Figure 6, in order to introduce how far the experimental data deviate from the optimum behavior determined by the analytical equations, as shown in Figure 7. Experimental data are lower than expected by analytical equations due to the experimental restrictions, such as measuring accuracy and thermal effects inside discharge chamber and plasma region. As well, the modeling process is based on sharply consecutive output data obtained from analytical equations, i.e., no deviation may be expected in the behavior of the modeling formulae. This may submit an advantage to those designing, operating or working on such systems to take into account the optimum behaviors extracted from similar experimental studies.

As the experimental data are generally lower than the modeling data, as shown in Figure 7, it looks that the variation of $p . d$ product versus distance $(d)$ is a characteristic feature-due to the identical behavior for both cases - of such systems. Hence, the experimental restrictions in such systems have to be carefully introduced and avoided — but definitely not omitted—at design course in order to approach the optimum parameters as possible.

Finally, the system considered in this work was used for deposition of metal oxide thin films (e.g., stainless steel films on glass substrates) and these films were featured by uniformity, very good adhesion and large area when compared to other deposition techniques available in the local research environment, therefore, these films could be described as high quality structures. However, the results of deposition were not included in this work as they are presented in other studies carried out using the same system.

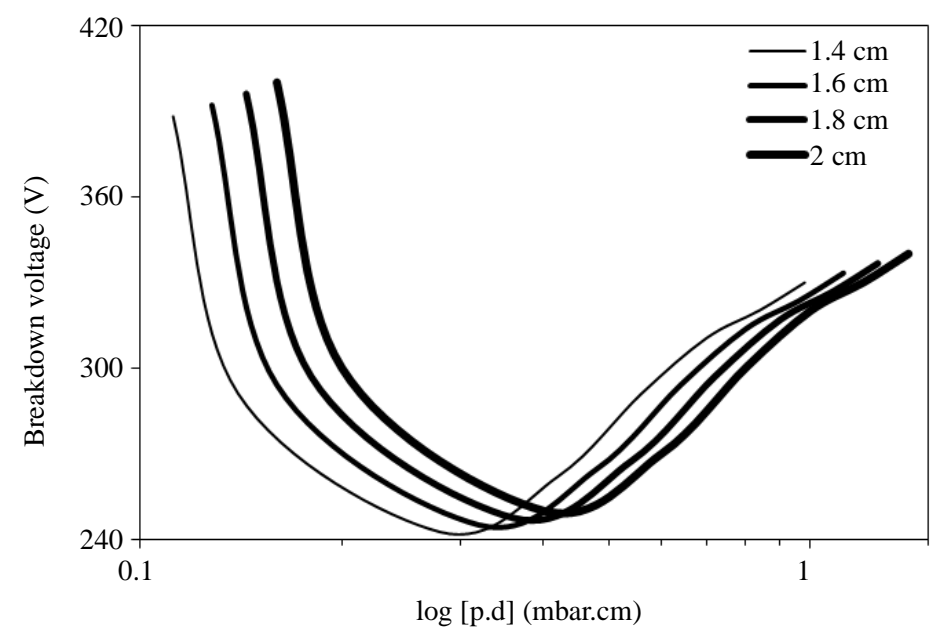

Figure 5. Paschen's curves for different inter-electrode spacing distances $(d<2 \mathrm{~cm})$ between the discharge electrodes.

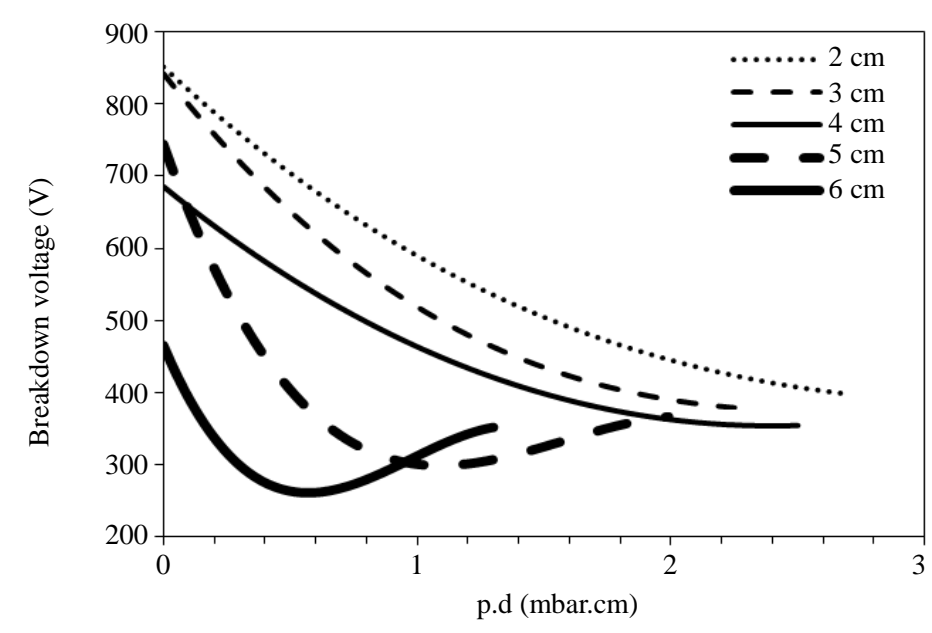

Figure 6. Modeling of Paschen's curves obtained at different interelectrode spacing distances. 


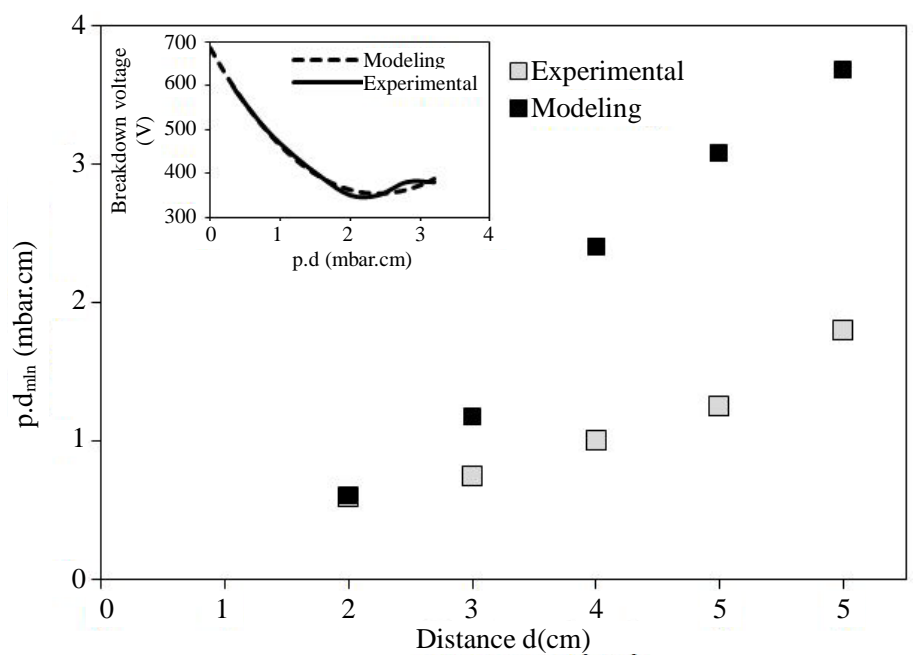

Figure 7. Comparison between experimental and modeling of minimum p.d product versus inter-electrode spacing distance $(d)$ (inset is the same comparison at distance of $4 \mathrm{~cm}$ ).

\section{Conclusion}

Referring to the results obtained from this work, the home-made dc magnetron plasma sputtering system was characterized to introduce its performance in accordance to Paschen's law and governing properties of such deposition systems. Results have showed that using magnetron at the cathode of discharge configuration highly has affected the relation of breakdown voltage to the gas pressure and distance product as the breakdown voltage was decreased and the minimum point was shifted to higher values of this product. Also, the characterization of Paschen's curve for this system in both experimental and modeling cases was introduced and showed that the experimental results are in agreement to those of modeling with some deviation due to the experimental restrictions included. As well, this system was found to satisfy the requirements for deposition of high-quality thin films from different materials.

\section{Acknowledgements}

Authors would like to thank Dr. Fuad T. Ibrahim at the same institution for his assistance to perform the machining work of the proposed design.

\section{References}

[1] McClanahan, E.D. and Laegreid, N. (1991) Production of Thin Films by Controlled Deposition of Sputtered Material. Topics in Applied Physics, 64, 339-377. http://dx.doi.org/10.1007/3540534288_21

[2] Rademacher, D., Zickenrott, T. and Vergöhl, M. (2013) Sputtering of Dielectric Single Layers by Metallic Mode Reactive Sputtering and Conventional Reactive Sputtering from Cylindrical Cathodes in a Sputter-Up Configuration. Thin Solid Films, 532, 98-105. http://dx.doi.org/10.1016/j.tsf.2012.11.101

[3] Baek, J.-S. and Kim, Y.J. (2006) Cooling Effect Enhancement in Magnetron Sputtering System. 5th International Conference on CFD in the Process Industries CSIRO, Melbourne, 13-15 December 2006, 1-5.

[4] Kelly, P.J. and Arnell, R.D. (2000) Magnetron Sputtering: A Review of Recent Developments and Applications. Vacuum, 56, 159-172. http://dx.doi.org/10.1016/S0042-207X(99)00189-X

[5] Arnell, R.D. and Kelly, P.J. (1999) Recent Advances in Magnetron Sputtering. Surface and Coatings Technology, 112, 170-176. http://dx.doi.org/10.1016/S0257-8972(98)00749-X

[6] Walkowicz, J., Zykov, A., Dudin, S. and Yakovin, S. (2006) ICP Enhanced Reactive Magnetron Sputtering System for Synthesis of Alumina Coatings. Tribologia, 6, 163-174.

[7] Ashenford, D.E., Long, F., Hagston, W.E., Lunn, B. and Matthews, A. (1999) Experimental and Theoretical Studies of the Low-Temperature Growth of Chromia and Alumina. Surface and Coatings Technology, 116-119, 699-704. http://dx.doi.org/10.1016/S0257-8972(99)00181-4 
[8] Sigmund, P. (1981) Topics in Applied Physics, Vol. 47. Sputtering by Particle Bombardment I. Springer-Verlag, Berlin.

[9] Taherkhani, F. and Taherkhani, A. (2010) Surface Characterization of Through Cage Plasma Nitriding on the Surface Properties of Low Alloy Steel. Transaction B: Mechanical Engineering, 17, 253-263.

[10] Wehner, G.K. and Anderson, G.S. (1983) Vacuum Technology, Thin Films and Sputtering: An Introduction. Academic Press, New York.

[11] Bogaerts, A., Neyts, E., Gijbels, R. and van der Mullen, J. (2002) Gas Discharge Plasmas and Their Applications (Review). Spectrochimica Acta Part B, 57, 609-658. http://dx.doi.org/10.1016/S0584-8547(01)00406-2

[12] Kotp, E.F. and Al-Ojeery, A.A. (2012) Studies the Effect of Magnetic Field on Argon Plasma Characteristics. Australian Journal of Basic and Applied Sciences, 6, 817-825.

[13] Personick, S.D. (1971) Statistics of a General Class of Avalanche Detectors with Applications to Optical Communications. Bell System Technical Journal, 50, 3075-3095. http://dx.doi.org/10.1002/j.1538-7305.1971.tb01847.x

[14] Wang, J.-C., Leoni, N., Birecki, H., Gila, O. and Kushner, M.J. (2013) Electron Current Extraction from Radio Frequency Excited Micro-Dielectric Barrier Discharges. Journal of Applied Physics, 113, Article ID: 033301.

[15] Window, B. and Savvides, N. (1986) Charged Particle Fluxes from Planar Magnetron Sputtering Sources. Journal of Vacuum Science \& Technology A, 4, 196. http://dx.doi.org/10.1116/1.573470

[16] Makabe, T. and Petrovic, Z. (2006) Plasma Electronics: Applications in Microelectronic Device Fabrication. Taylor \& Francis, New York, 301. 